ZENO, Institute of Philosophy

Logic GroupPreprint Series

No. 199- revised May 2000

\title{
Arguments for the Continuity Principle
}

M. van Atten and D. van Dalen ${ }^{1}$

\section{Contents}

1 The continuity principle 1

2 A phenomenological consideration $\quad 8$

2.1 An argument for $\mathrm{G}(\mathrm{raph}) \mathrm{WC}-\mathrm{N} \ldots \ldots . . \ldots 8$

2.2 Two arguments against WC-N . . . . . . . . . . 13

3 Other arguments for continuity $\mathbf{1 5}$

3.1 Undecidability of equality of choice sequences . . . . . . 15

3.2 Kripke's Schema and full PEM . . . . . . . . . . . . 15

3.3 The KLST theorem ................ 16

4 Conclusion

\section{The continuity principle}

There are two principles that lend Brouwer's mathematics the extra power beyond arithmetic. Both are presented in Brouwer's writings with little or no argument. One, the principle of bar induction, will not concern us here. The other, the continuity principle for numbers, occurs for the first time in print in [Brouwer 1918]. It is formulated and immediately applied to show that the set of numerical choice sequences is not enumerable. In fact, the idea of continuity can be dated fairly precisely, it is inserted in the margin of Brouwer's notes for his course on Pointset Theory of 1915/16. The course was given again in 1916/17 and he must have inserted it as new material right at the beginning.

In modern language, the principle reads

\footnotetext{
${ }^{1}$ Department of Philosophy, Utrecht University, Heidelberglaan 8, NL-3584 CS Utrecht, The Netherlands. Email: mark.vanatten@phil.uu.nl, dirk.vandalen@phil.uu.nl. We wish to thank Anne Troelstra and Albert Visser for their comments, which led to several improvements.
} 


$$
\text { (WC-N) } \forall \alpha \exists x A(\alpha, x) \Rightarrow \forall \alpha \exists m \exists x \forall \beta[\bar{\beta} m=\bar{\alpha} m \rightarrow A(\beta, x)]
$$

where $\alpha$ and $\beta$ range over choice sequences of natural numbers, $m$ and $x$ over natural numbers, and $\bar{\alpha} m$ stands for $\langle\alpha(0), \alpha(1), \ldots, \alpha(m-1)\rangle$, the initial segment of $\alpha$ of length $m$.

An immediate consequence of WC-N is that all full functions are continuous, and, as a corrolary, that the continuum is unsplittable [Veldman 1982]. Note that it is also incompatible with Church's thesis [Troelstra-Dalen 1988], section 4.6.

After Brouwer, Troelstra was the first to ask in print for a conceptual motivation, but he remained an exception, most authors followed Brouwer by simply accepting it.

Let us note first that in one particular case the principle is obvious indeed, namely in the case of the lawless sequences. The notion of lawless sequence surfaced fairly late in the history of intuitionism. Kreisel introduced it in [Kreisel 1968] for metamathematical purposes. There is a letter from Brouwer to Heyting in which the phenomenon also occurs [Brouwer 1942]. This is an important and interesting fact since it is (probably) the only time that Brouwer made use of a possibility expressly stipulated in, e.g., [Brouwer 1925].

For Brouwer the universe of choice sequences was of a rich variety, it contained both 'more or less arbitrarily chosen sequences' and lawless sequences. He envisaged, so to speak, a continuous spectrum of freedom of choice, running from completely free to completely determined. Kreisel, following a suggestion by Gödel, introduced the term 'lawless' for 'completely free'. A lawless sequence is a sequence of natural numbers where at each choice no restrictions are made with respect to future choices. Because so little is known at each moment about such a sequence, one can defend an even stronger principle for them:

$$
\text { (open data) } A(\alpha) \Rightarrow \exists x \forall \beta \in \bar{\alpha} x A(\beta)
$$

( $\beta \in \bar{\alpha} x$ means that $\beta$ shares with $\alpha$ an initial segment of length $x$.) Let us just go through the justification for this principle. Suppose we know $A(\alpha)$, i.e., we have a proof for it, then the proof must be given in a finite time. But at the moment that proof is completed only a finite number of outputs of $\alpha$ have been produced, say, $\alpha(0), \ldots, \alpha(k-1)$. Now the future of $\alpha$ after choice $\alpha(k-1)$ is open, i.e., the proof can only depend on $\bar{\alpha} k$. But then it is clear that $A$ holds for any continuation of $\bar{\alpha} k$. This principle evidently fails for the class of all choice sequences, since $\alpha$ could be lawlike, say constant. In that case the principle of open data would not apply to 
$\exists y \forall x(\alpha(x)=y)$.

So the class of all choice sequences asks for another principle. It is historically interesting that Brouwer avoided the trap suggested by the lawless sequences, his formulation of the continuity principle was right the first time. Hermann Weyl seems to have fallen into the trap, although it must be added that the formulations in Weyl's 'Grundlagenkrise' paper, [Weyl 1921], leave room for other interpretations. These issues are discussed in [Dalen, D. van 1995] and [Atten, M. van 1999].

Brouwer's correct formulation of the continuity principle makes it very plausible that he had varying restrictions, restrictions on restrictions, etc., in mind (see below). The later explicit formulation of 'choice sequence' therefore looks more like a forgotten clause than a new idea.

A simple minded argument for the continuity principle could run as follows:

Suppose we have a proof of $\forall \alpha \exists x(F(\alpha)=x)$, where $F$ is a function.

Now pick an $\alpha$ and start making the proof instantiation for $\exists x(F(\alpha)=x)$, i.e., we construct a number $n$ and a proof of $F(\alpha)=n$. When this construction is finished, only finitely many outputs of $\alpha$ have been chosen, and therefore any $\beta$ with the same initial segment satisfies $F(\beta)=n$.

In order to illustrate the gap in the argument let us consider a simple example.

Imagine the following game between two players, $A$ and $B$. $A$ generates a choice sequence $\alpha$ and $B$ has a procedure to calculate $F(\alpha)$. For the sake of the example, assume that $B$ will assign to any $\alpha$ the number $\alpha(100)$. Now the game runs as follows: at each move $A$ gives information about $\alpha, B$ looks at it and produces the output (telling $A$ that he may stop), or asks for another bit of information.

Here is a brief run:

\begin{tabular}{cc}
$A$ & $B$ \\
\hline$\alpha(0)=1$ & $?$ \\
$\alpha(1)=7$ & $?$ \\
$\alpha(2)=0$ & $?$ \\
$\alpha(3)=2$ and & $F(\alpha)=2$
\end{tabular}

from now on $\alpha$ is constant. 
So only 4 outputs of $\alpha$ are required to determine $F(\alpha)$. However, it is by no means the case that $F(\beta)=2$ for any $\beta$ with initial segment $\langle 1,7,0,2\rangle$.

The error is caused by the fact that $A$ suddenly produces information of a higher order. The argument could therefore be patched up by stipulating that only numerical information may be passed on.

That would, however, not be acceptable at all. It would simply mean that within certain limits (to be precise, the spread conditions) we would act as if all sequences were lawless, and hence establish the principle for that class. But that was not the problem - we wanted to establish the principle for all (or maybe a suitable class of) choice sequences.

If we inspect Brouwer's introduction of choice sequences, we see that he realized the difficulties involved in allowing non-lawlike objects. In his first public presentation he hardly paid attention to the sequences, but rather concentrated on laying down the restictions on numerical choices. This was done in the definition of a spread (Menge), [Brouwer 1918], pp.2-3:

A spread is a law on the basis of which, if again and again an arbitrary complex of digits [a natural number] of the sequence $\zeta$ [the natural number sequence] is chosen, each of these choices either generates a definite symbol, or nothing, or brings about the inhibition of the process together with the definitive annihilation of its result; for every $n$, after every uninhibited sequence of $n-$ 1 choices, at least one complex of digits can be specified that, if chosen as $n$-th complex of digits, does not bring about the inhibition of the process. Every sequence of symbols generated from the spread in this manner (which therefore is generally not representable in finished form) is called an element of the spread. We shall also speak of the common mode of formation of the elements of a spread M as, for short, the spread M. ${ }^{2}$

\footnotetext{
${ }^{2}$ The original reads:
}

Eine Menge ist ein Gesetz, auf Grund dessen, wenn immer wieder ein willkürlicher Ziffernkomplex der Folge $\zeta$ gewählt wird, jede dieser Wahlen entweder ein bestimmtes Zeichen, oder nichts erzeugt, oder aber die Hemmung des Prozesses und die definitive Vernichtung seines Resultates herbeiführt, wobei für jedes $n$ nach jeder ungehemmten Folge von $n-1$ Wahlen wenigstens ein Ziffernkomplex angegeben werden kann, der, wenn er als $n$-ter Ziffernkomplex gewählt wird, nicht die Hemmung des Prozesses herbeiführt. Jede in dieser Weise von der Menge erzeugte Zeichenfolge (welche also im allgemeinen nicht fertig darstellbar ist) heisst ein Element der Menge. Die gemeinsame Entstehungsart der Elemente einer Menge $M$ werden wir ebenfalls kurz als die Menge M bezeichnen. 
In modern language, the spread law determines a decidable subtree of the tree of all finite sequences of natural numbers satisfying a simple basic condition:

For each finite sequence $\left\langle a_{0}, a_{1}, a_{2}, \ldots a_{n}\right\rangle$ in the subtree, there is an immediate extension $\left\langle a_{0}, a_{1}, a_{2}, \ldots a_{n}, a_{n+1}\right\rangle$ which is also in the subtree.

This condition ensures that there are no finite maximal paths in the subtree. The infinite paths are called choice sequences; they are the elements of the spread. No further explanation of the notion of choice sequences is given.

In the next major exposition, [Brouwer 1925], Brouwer explicitly allowed over and above the spread condition, restrictions of future choices; in footnote 3 (p.245) he mentions that he thinks of the elements of a spread as 'including the characteristic of their freedom of continuation, which after each choice can be limited arbitrarily (possibly to complete determination, in any case according to a spread law) ${ }^{\prime}{ }^{3}$ In a later, handwritten, note in the margin of one of his reprints, he added

The arbitrariness of this, under preservation of the possibility of extension, of the 'restriction condition' associated with of a finite choice sequence, lends that choice sequence, and hence also all its extensions, a new arbitrariness. In the spread also an arbitrary well-ordered set of these restriction conditions can be assigned (where thus, for example, a restriction of the restrictions for the following choices of a finite choice sequence may be assigned). ${ }^{4}$

In [Brouwer 1942] he returned to this particular footnote with the words

[Brouwer 1947] contains the following clarifying remark on this definition:

Because mathematics is independent of language, the word symbol (Zeichen) and in particular the words complex of digits (Ziffernkomplex) must be understood in this definition in the sense of mental symbols, consisting in previously obtained mathematical concepts.

${ }^{3}$ Inklusive des Charakters ihrer Fortsetzbarkeitsfreiheit, welche sich nach jeder Wahl beliebig (eventuell bis zur völligen Bestimmtheit, jedenfalls aber einem Mengengesetze entsprechend) verengern kann.

${ }^{4}$ Die Beliebigkeit dieser unter Erhaltung, der Fortsetzbarkeitsmöglichkeit einer endlichen Wahlfolge zugeordneten 'Verallgemeinerungszusatzes' erteilt dieser Wahlfolge, mithin auch ihren Fortzetzungen, eine neue Willkür. Von derartigen Verengerungszusätze kann nun in der Menge auch eine beliebige wohlgeordnete Spezies angebracht werden (wobei also einer endlichen Wahlfolge z.B. eine Verengerung der für die weiteren Wahlen bestehenden Verengerungszusatzfreiheit zugeordnet werden kann). 
The somewhat brief footnote on the definition of the spread element might gain more clarity in the following more elaborated version:

The freedom of extension of a sequence of series of signs, generated by an unbounded choice sequence, which is an element of the representing spread, can furthermore after each choice be restricted arbitrarily (e.g., to complete determination, or also according to a spread law). And indeed the arbitrariness of the assignment of restriction conditions to individual choices under preservation of the possibility of extension, forms an essential characteristic of the free growing of the spread element. Each single restriction condition can again be assigned a restriction condition of second order, which restricts the arbitrariness of future restriction conditions, etc. ${ }^{5}$

Hence Brouwer did - at that time - intend to incorporate higher order restrictions. The practical significance of such refinements is not immediately clear. Indeed, in mathematical practice, one usually does not require much more than knowledge of the spread law, and possibly the presence of lawlike sequences. The latter are required to instantiate specific existence claims.

From the viewpoint of metamathematics the lawless sequences are of considerable importance, cf. [Kreisel 1958], [Troelstra-Dalen 1988]. They provide the simplest example of a class of choice sequences governed by a higher order restriction, namely the demand that all (first-order) restrictions on the numerical choices will be trivial, i.e., at each stage all numbers are eligible.

For actual mathematics they are, however, not suitable, since they defy even the simplest closure properties. Therefore a more liberal class of choice sequences has to be considered. A general schema for choice sequences looks as follows.

The subject successively chooses objects (say natural numbers), restrictions on future choices, restrictions on restrictions of future choices, etc.

\footnotetext{
${ }^{5}$ Die etwas kurz gehaltene Fussnote zur Definition des Mengenelementes dürfte in der folgenden ausführlicheren Fassung an Deutlichkeit gewinnen:

Die Fortsetzbarkeitsfreiheit einer von einer unbegrenzten Wahlfolge erzeugten, ein Element der Menge darstellenden Folge von Zeichenreihen kann übrigens nach jeder Wahl beliebig (z.B. bis zur völligen Bestimmtheit, oder auch einem Mengengesetze entsprechend) verengert werden, und zwar stellt die Beliebigkeit dieser den einzelnen Wahlen unter Erhaltung der Fortsetzbarkeitsmöglichkeit zuzuordnenden Verengerungszusätze einen wesentlichen Charakter des freien Werdens des Mengenelementes dar. Jedem einzelnen Verengerungszusatz kann wieder ein die Beliebigkeit der weiteren Verengerungszusätze einschränkender Verengerungszusatz zweiter Ordnung beigegeben werden, usw.
} 
These lists, of course, have to terminate. So a choice sequence written in full generality looks like

$\left(n_{0}, R_{0}^{0}, R_{1}^{0}, \ldots R_{k_{0}}^{0}\right),\left(n_{1}, R_{0}^{1}, R_{1}^{1}, \ldots R_{k_{1}}^{1}\right), \ldots$ This agrees with Brouwer's verbal specifications.

Later in life Brouwer started to have qualms about higher order restrictions. In [Brouwer 1981], he provided a footnote to the definition of choice sequence, stating that

In some former publications of the author, restrictions of freedom of future restrictions of freedom, restrictions of restricions of freedom of future restrictions of freedom, and so on, were also admitted. But at present the author is inclined to think this admission superfluous and perhaps leading to needless complications.

All he allowed was 'Finally the freedom of proceeding, without being completely abolished, may at some $p_{\nu}$ [stage] undergo some restriction, and later on further restrictions.'

In [Brouwer 1952], he even went so far as to withdraw his earlier views altogether: 'In former publications I have sometimes admitted restrictions of freedom. However, this admission is not justified by close introspection, and moreover would endanger the simplicity and rigour of further developments.' Heyting, quite sensibly, added the comment that 'It is not clear how introspection could forbid us to introduce the notion of second order restriction. The reason for not introducing them is simply that they are hard to manage.', [Brouwer 1975], p.607.

One might put forward another reason: for the general notion (with full restrictions), it is not clear how to justify the continuity principle. In fact it is not hard to give an example of a class of choice sequences for which the continuity principle does not hold. Consider the full tree of all finite sequences of natural numbers. We will allow choice sequences with first and second order restrictions: there is one constant second-order restriction which says that all first-order restrictions are of the form 'after a finite number of choices the sequence becomes constant'. So each first-order restriction can be written as a finite sequence $\left(n_{0}, n_{1} \ldots, n_{k}\right)$, where $\alpha(0)=n_{0}, \alpha(1)=n_{1}, \ldots, \alpha(k)=n_{k}$, and $\forall p>k\left(\alpha(p)=n_{k}\right)$. This yields perfectly good choice sequences according to the liberal definition. Now we define a function on this set of choice sequences as follows: $F(\alpha)=n_{k}$, where $\alpha$ is given by the above description. $F$, clearly, is not continuous!

Note also that the class of all primitive recursive functions falls under the liberal schema of Brouwer.

In spite of the danger of complications, including the loss of the continuity principle, higher order restrictions are useful, not a priori excluded, and 
perhaps necessary. We wish, however, to retain the continuity principle, the prime instrument of the theory of choice sequences. So, here is a topic for research: find suitable conditions for classes of choice sequences, so that we will be able to use the continuity principle. And, parallel to that, give a justification for the continuity principle that has the widest possible range of application.

\section{A phenomenological consideration}

\subsection{An argument for $\mathrm{G}$ (raph) WC-N}

As Troelstra (e.g., [Troelstra 1977], p.151, [Troelstra 1983]) has noted, justifications of WC-N so far have not gone beyond plausibility considerations. In this section, we want to give an informal, rigorous ${ }^{6}$ derivation of a restricted weak continuity principle. This restricted principle suffices for the intuitionistic reconstruction of analysis, Brouwer's original motivation for the introduction of choice sequences. ${ }^{7}$ In the following formulation, the predicate refers only to the graph of $\alpha$.

$$
\text { (GWC-N) } \forall \alpha \exists x A(\alpha, x) \Rightarrow \forall \alpha \exists m \exists x \forall \beta[\bar{\beta} m=\bar{\alpha} m \rightarrow A(\beta, x)]
$$

Some comments on the condition on $A$ are in order. Troelstra has pointed out (in [Troelstra 1985] and in personal communication) that in the case of choice sequences, one can speak of two concepts of extensionality.

First, there is the usual, classical concept, according to which a predicate $A(\alpha, x)$ is extensional if it satisfies

$$
\alpha=\beta \wedge x=y \wedge A(\alpha, x) \rightarrow A(\beta, y)
$$

Second, there is the stronger concept according to which in the definition of $A(\alpha, x), \alpha$ is referred to only through its graph.

We will refer to the first concept by it usual name, 'extensionality', and to the second by 'graph-extensionality'.

Clearly graph-extensionality implies extensionality, but the converse does not hold. The reason is that, for incomplete objects, in general the only way to know that $\alpha=\beta$ is to know that they are generated in the very

\footnotetext{
${ }^{6}$ For the notion of 'informal rigour', see Kreisel [Kreisel 1967] and Troelstra [Troelstra 1985].

${ }^{7}$ It conforms to the use Brouwer makes of his continuity principle, and also to [Veldman 1982]. The phenomenological details of how the nature of the continuum motivates the introduction of choice sequences are spelled out in [Atten, M. van 1999].
} 
same process. But this opens the door to referring to other information than just the graph. Examples are [Troelstra 1985], pp.221-222, and Visser's proof below, section 2.2, that the present argument does not work for all extensional predicates. The latter example explains the restriction on $A$.

The argument in the present section was arrived at in an attempt to apply Husserl's principle of the noetic-noematic correlation [Husserl 1976], section 93, to choice sequences. Roughly, this principle states that the structure of the way an object is given to us is parallel to the structure of the acts in which that object is intended. In the case of choice sequences, this led to the question: in what ways can the freedom the subject enjoys in the process of generation be reflected in the intensional properties of the sequences themselves? That suggested the idea of provisional restrictions (below), and also the already familiar concepts of lawlike and lawless sequences. It is important to keep in mind that choice sequences are given to us as individual objects. $^{8}$

Let us recall the arbitrariness in posing restrictions, as stressed by Brouwer in the quotes above, see p.5. This essential freedom motivates the following division of restrictions in two kinds, which we will call 'provisional' and 'definitive'. The subject can make any revision in its ideas about how to go on generating a choice sequence as long as

1. the revision does not go against any other restrictions that are already in effect,

2. the revision admits the existing initial segment,

3. after the revision, it is still possible to extend any admissible initial segment.

Thus we have

definitive restrictions which have the form 'from now on, restriction $R_{k}^{i}$ holds, and it will not be revised anymore' (with or without a specified initial segment);

provisional restrictions which have the form 'for an unspecified number of stages, restriction $R_{k}^{i}$ holds'.

As long a restriction on a particular sequence is not definitive, that restriction can be reconsidered at some (or any) stage. (Because it is up to the subject's own choice to make a restriction either provisional or definitive, what kind it is dealing with is decidable.) A provisional restriction, in

${ }^{8}$ Following Troelstra's 'analytic approach', e.g., [Troelstra 1983]. 
spite of its uncommitted character, is a genuine restriction: without lifting it first, one cannot make a choice that does not accord with it - on pain of inconsistent behaviour.

If several restrictions are in effect, their conjunction counts as provisional exactly if at least one of its members is. Note that, for an individual choice sequence to be given to us, it is not required that any of its restrictions is definitive.

As choice sequences are generated by the subject, imposed restrictions correspond to intensional properties of the sequence. The distinction between provisional and definitive restrictions therefore induces a corresponding distinction between provisional and definitive intensional properties. In phenomenological argot, 'restriction' stresses a noetic, 'property' a noematic aspect of the constitution of a choice sequence.

On the other hand, not every intensional property corresponds to a restriction. There are other intensional aspects as well, such as the order of generation of the sequence. This is exploited in the arguments in section 2.2.

An example of an already known concept of choice sequence that could be interpreted as an implicit application of the provisional-definitive distinction, are the hesitant sequences in [Troelstra-Dalen 1988], p.208:

A hesitant sequence (say $\beta$ ) is a process of generating values $\beta 0, \beta 1, \beta 2, \ldots \in \mathbb{N}$ such that at any stage we either decide that henceforth we are going to conform to a law in determining future values, or, if we have not already decided to conform to a law at an earlier stage, we freely choose a new value of $\beta[\ldots]$ $[\mathrm{T}]$ he decision whether or not to conform to a law may stay open indefinitely.

Thus, we can say that a hesitant sequence is a provisionally lawless sequence (which at any time may be turned into a lawlike sequence).

Let us see how the existence of this distinction justifies GWC-N. We will first consider the case of the universal spread, and then generalize the argument to arbitrary spreads.

The universal spread $C$ is the spread in which each finite sequence $\left\langle a_{0}, a_{1}, a_{2}, \ldots a_{n}\right\rangle$ may be extended by any number $a_{n+1}$. $C$ is the universal tree over $\mathbb{N}$.

On p.253 of [Brouwer 1925], Brouwer formulates the following continuity principle for $C$ :

A law that assigns to each element $g$ of $C$ an element $h$ of $A$ [the natural numbers], must have determined the element $h$ completely after a certain initial segment $\alpha$ of the sequence of numbers 
of $g$ has become known. But then to every element of $C$ that has

$\alpha$ as an initial segment, the same element $h$ of $A$ will be assigned. ${ }^{9}$

The assignment of a number to a choice sequence requires a construction. At the time of construction, only an initial segment of the sequence is known, as well as some intensional properties.

If all intensional properties referred to are restrictions, the intensional properties may be provisional and therefore open to revision. In this setting, the intensional properties that a sequence has at any particular stage do not necessarily characterize that sequence also at all later stages. That in turn means that a construction that depends on these intensional properties may yield different results at different stages. However, it is understood that once we have a proof that a relation $A(\alpha, x)$ holds between a choice sequence and a number, this proof remains valid once and for all. The problem with a construction that depends on provisional properties is that it cannot guarantee this lasting validity: should the provisional properties change, the outcome of the construction might as well. Therefore such a construction cannot count as a proof of $A(\alpha, x)$.

To illustrate this, let us look at a previous example (p.3) again. Suppose we have, of a choice sequence $\alpha$, the initial segment $\langle 1,7,0,2\rangle$, and the definitive restriction 'From now on, $\alpha$ is constant'. From this information, we can immediately conclude that $\alpha(100)$ will be 2 . Now suppose we have, of a choice sequence $\beta$, the initial segment $\langle 1,7,0,2\rangle$, and the provisional restriction 'From now on, $\beta$ is constant'. In this case we cannot conclude that $\beta(100)$ will be 2 . Maybe we decide to lift the provisional restriction somewhere after our fourth choice; if we do so, we can then consistently choose $\beta(100)$ different from 2.

Earlier on we put the condition on $A(\alpha, x)$ that of $\alpha$, only the graph is referred to. That allows us to say now that any intensional property that might be useful in constructing an $x$ such that $A(\alpha, x)$, should already be extractable from the first-order restrictions, as only these bear directly on the graph of $\alpha$. But we just saw that, if we allow provisionality, restrictions cannot be depended on when constructing such an $x$. Hence the construction can only depend on the other information that we have, given the restriction on $A$. This other information, of course, is just the initial segment. But that is precisely what GWC-N says. ${ }^{10}$

\footnotetext{
${ }^{9}$ Ein Gesetz, das jedem Elemente $g$ von $C$ ein Element $h$ von $A$ [the natural numbers] zuordnet, muß nämlich das Element $h$ vollständig bestimmt haben nach dem Bekanntwerden eines gewissen Anfangssegmentes $\alpha$ der Nummernfolge von $g$. Dann aber wird jedem Elemente von $C$, das $\alpha$ als Anfangssegment besitzt, dasselbe Element $h$ vom $A$ zugeordnet.

${ }^{10}$ Note that, because of the condition on $A$, the presence (or absence) of provisional restrictions on $\alpha$ cannot be exploited in the definition of $A$.
} 
This argument requires that the spread contains, for each element $\alpha$, the 'provisionalized' version $\alpha$ ' as well. 'Provisionalized' means that $\alpha$ and $\alpha^{\prime}$ have the same initial segment and are subject to the same restrictions, but in the case of $\alpha^{\prime}$ all of these restrictions are provisional. (Hence $\alpha$ and $\alpha^{\prime}$ are not necessarily distinct.) In $C$, which admits all choice sequences, this requirement is certainly met; hence we conclude that GWC-N holds for $C$.

In [Brouwer 1927], p.63, Brouwer extended the formulation of the continuity principle for $C$ to arbitrary spreads (i.e., subspreads of $C$ ):

Let $M$ be an arbitrary spread, let $\mu$ be the denumerably infinite spread of finite (inhibited or uninibited) choice sequences $F_{s n_{1} \ldots n_{r}}$ upon which $M$ is based (where $s$ and the $n_{\nu}$ represent the natural numbers chosen oen after the other for the choice sequence in question), and let the natural number $\beta$ be associated with each element of $M$. Then there is distinguished in $\mu$ a removable numerable subspread $\mu_{1}$ of uninhibited finite choice sequences such that with an arbitrary element of $\mu_{1}$ the same natural number $\beta$ is associated for all elements of $M$ issuing from $\mu_{1}$, while furthermore a proof $h$ is given that makes it apparent, for an arbitrary uninhibited element of $\mu$, that every uninhibited infinite choice sequence issuing from it possesses an [initial] segment belonging to $\mu_{1} \cdot 11$

To obtain GWC-N in this case, we resort to a trick. The way to define a subspread in effect consists in specifying which choice sequences are to be removed from $\mathrm{C}$; for some $m$, their initial segments of length $\geq m$ are inhibited. Hence to these sequences no value will be assigned. But we can embed the subspread into the universal spread if, instead of inhibiting any

\footnotetext{
${ }^{11}$ The translation is adapted from the English version of [Brouwer 1927], p.63, in [Heijenoort, J. van 1967], p.459.

Sei $M$ eine beliebige Menge, $\mu$ die ihr zugrunde liegende abzählbar unendliche Menge der endlichen (gehemmten und ungehemmten) Wahlfolgen $F_{s n_{1} \ldots n_{r}}$ (wo $s$ und die $n_{\nu}$ die für die betreffende Wahlfolge der Reihe nach gewählten natürlichen Zahlen vorstellen), und sei jedem Elemente von $M$ eine natürliche Zahl $\beta$ zugeordnet. Alsdann ist in $\mu$ eine solche abzählbare Teilmenge $\mu_{1}$ von ungehemmten endlichen Wahlfolgen ausgezeichnet, daß einem beliebigen Elemente von $\mu_{1}$ für alle aus ihm hervorgehenden Elemente von $M$ dieselbe natürliche Zahl $\beta$ zugeordnet ist, während weiter eine Beweisführung $h$ vorliegt, mittels welcher sich für ein beliebiges ungehemmtes Element von $\mu$ herausstellt, daß jede aus ihm hervorgehende ungehemmte unendliche Wahlfolge zu einen zu $\mu_{1}$ gehörigen Abschnitt besitzt.
} 
sequences, we assign a default value to them. (Note that in Brouwer's conception of mathematical entities as generated by the subject, whether or not a sequence is inhibited is decidable.) This embedding enables us to apply the argument for $C$ again, thus showing GWC-N for the subspread as well. ${ }^{12}$

\subsection{Two arguments against WC-N}

The restriction, in the argument in section 2.1, that $A$ be graph-extensional, is necessary, as shown by the following

Theorem Assume the creating subject generates choice sequences as individual objects, and can therefore enumerate the sequences generated so far. Then WC-N does not hold generally for extensional predicates.

We have two proofs, the second one actually yielding a stronger result.

First proof. Assume we have an operation $F$ (not necessarily a function) that enumerates the choice sequences. Then

$$
\forall \alpha \exists n(\alpha=F(n))
$$

Put $G(\alpha, n) \equiv \alpha=F(n)$ to get

$$
\forall \alpha \exists n G(\alpha, n)
$$

and apply WC-N. This yields

$$
\forall \alpha \exists m \exists n \forall \beta[\bar{\beta} m=\bar{\alpha} m \rightarrow G(\beta, n)]
$$

But this means that the same $n$ will be paired to different choice sequences, which conflicts with the notion of 'enumeration'. We conclude that in the presence of an enumeration, WC-N does not hold generally. (Note that the predicate $G$ is not graph-extensional.)

There is also WC-N!, the 'functional' version of $\mathrm{WC}-\mathrm{N}:{ }^{13}$

\footnotetext{
${ }^{12}$ The following suggestion is due to Troelstra. It may be possible to model provisional conditions mathematically by projections of lawless sequences, along the following lines. Assume the possible restrictions are enumerable. Think of the generation process of a choice sequence as the choosing of triples $\left(n_{0}, R_{0}, t_{0}\right),\left(n_{1}, R_{1}, t_{1}\right),\left(n_{2}, R_{2}, t_{2}\right), \ldots$ Here, $n_{i}$ is the number chosen at stage $i, R_{i}$ is a (provisional) restriction, and $t_{i}$ is a sequence of integers all $\leq i$ in absolute value. If $+j$ appears in $t_{i}$, this means that restriction $R_{j}$ is made definitive; $-j$ means that restriction $R_{j}$ is lifted. Within this setting, we may consider the sequence of triples as lawless; what is projected from it is a choice sequence with variable second-order restrictions. No mathematical theory has been developed for those yet. (For the modelling of choice sequences with only (definitive) first-order restrictions by projections, see [Troelstra-Dalen 1970] and [Hoeven, G. van der 1981].)

${ }^{13}$ Similarly, GWC-N has a functional version GWC-N!, which seems to be the principle that Brouwer actually used.
} 
(WC-N!) $\forall \alpha \exists ! x A(\alpha, x) \Rightarrow \forall \alpha \exists m \exists x \forall \beta[\bar{\beta} m=\bar{\alpha} m \rightarrow A(\beta, x)]$

The second proof, due to Albert Visser, shows that neither WC-N nor WC-N! holds generally. It is an adaptation of an ingenious trick that Michael Beeson came up with in a recursion-theoretic context [Beeson 1975]. The idea is to construct an extensional predicate based on an intensional property of choice sequences.

Second proof. Let us introduce some notation:

1. $\mathbf{0}=\lambda x .0$, i.e. the constant zero sequence.

2. $P(n) \equiv \overline{\alpha_{n}}(n)=\overline{\mathbf{0}}(n) \vee \exists s<n\left[\alpha(s) \neq 0 \wedge \overline{\alpha_{n}}(s)=\overline{\mathbf{0}}(s) \wedge\right.$ $\left.\exists k \leq s\left(\overline{\alpha_{k}}(s+1)=\bar{\alpha}(s+1)\right)\right]$

3. $\mathcal{B}\left(\alpha_{n}\right)=\left\{\begin{array}{lll}0 & \text { if } & P(n) \\ 1 & \text { if } & \neg P(n)\end{array}\right.$

Here $\mathcal{B}$ is Beeson's functional, cf. [Beeson 1985], p. 62.

Claim 1: $\mathcal{B}$ is extensional.

Assume $\alpha_{n}=\alpha_{m}$ and $\mathcal{B}\left(\alpha_{n}\right)=0$. If the first $n$ values of $\alpha$ are 0 , then since $\alpha_{n}$ and $\alpha_{m}$ are are extensionally equal, the two cases, (i) $m<n$ and (ii) $m>n$, both yield $\mathcal{B}\left(\alpha_{m}\right)=0$; (i) is obvious, and for (ii) the second part of the clause of $P(m)$ trivially holds.

If $\mathcal{B}\left(\alpha_{n}\right)=0$ on the basis of the second clause of $P(n)$, then there exists an $s<n$ such that $\alpha_{n}(s) \neq 0$ and $\exists k \leq s\left(\overline{\alpha_{k}}(s+1)=\overline{\alpha_{n}}(s+1)\right)$. Now the two cases are (i) $m<s$ and (ii) $m \geq s$. Both cases immediately give $\mathcal{B}\left(\alpha_{m}\right)=0$.

Claim 2: $\mathcal{B}$ is discontinuous in $\mathbf{0}$.

Consider an initial segment $\overline{\mathbf{0}}(k)$ and assume that $\overline{\alpha_{n}}(k)=\overline{\mathbf{0}}(k) \rightarrow \mathcal{B}\left(\alpha_{n}\right)=0$ for all $n$. Now consider the finitely many $\alpha_{p}$ with $p \leq k$ and $\overline{\alpha_{p}}(k)=\overline{\mathbf{0}}(k)$, let $a=\max \left\{\alpha_{p}(k)+1 \mid p \leq k \wedge \overline{\alpha_{p}}(k)=\overline{\mathbf{0}}(k)\right\}$. Pick an $q>a$ such that $\alpha_{q}(k)=a$ and $\overline{\alpha_{q}}(k)=\overline{\mathbf{0}}(k)$, then by definition $\mathcal{B}\left(\alpha_{q}\right)=1$.

Claim 3: $\mathcal{B}$ depends on the enumeration.

This can be seen by making a suitable modification of a given enumeration.

Now we are almost done, put $\Phi(\alpha, x) \equiv \exists n\left(\alpha=\alpha_{n} \wedge \mathcal{B}\left(\alpha_{n}\right)=x\right.$. We see that $\forall \alpha \exists x \Phi(\alpha, x)$, and now the first proof shows that weak continuity fails 
for $\Phi$.

This second proof is easily generalized to prove the following theorem: for enumerable sets of choice sequences that contains a limit point, neither WC-N nor WC-N! holds generally.

\section{Other arguments for continuity}

\subsection{Undecidability of equality of choice sequences}

Let $f: \mathbb{N}^{\mathbb{N}} \rightarrow \mathbb{N}$ be discontinuous in the sense that a point of positive discontinuity is given. It is no restriction to assume that $f$ is discontinuous in $\mathbf{0}$, and that moreover $f(\mathbf{0})=0$. So we have $\forall n \exists \alpha \in \overline{\mathbf{0}}(n)(f(\alpha) \neq 0)$. Let us call the $\alpha$ associated to $n \alpha_{n}$. Geometrically speaking, we have the leftmost branch $\mathbf{0}$ in the underlying tree with a denumerable sequence of side branches $\alpha_{n}$, which all produce non-zero outputs under $f$. It is no restriction to assume that $\alpha_{n+1}$ branches off later than $\alpha_{n}$.

The nodes of all the $\alpha_{n}$ determine a subspread $S$. We define a mapping $g$ of $\mathbb{N}^{\mathbb{N}}$ onto $S$ by first giving a mapping of the full tree onto the underlying tree of $S$ : each node $\vec{n}$ is taken to the rightmost node $\vec{m}$ on $S$ with the same length. This mapping of nodes induces a unique mapping $g: \mathbb{N}^{\mathbb{N}} \rightarrow S$.

We now see that $\forall \alpha(\alpha \# \mathbf{0} \leftrightarrow f(g(\alpha)) \neq 0)$, and so $\forall \alpha(\alpha=\mathbf{0} \leftrightarrow f(g(\alpha))=0)$; hence we get $\forall \alpha(\alpha=\mathbf{0} \vee \alpha \neq \mathbf{0})$. Now we may consider our job done, as it is not decidable whether a choice sequence is the constant zero sequence or not.

We may also invoke the homogeneity of $\mathbb{N}^{\mathbb{N}}$ and conclude $\forall \alpha \forall \beta(\alpha=\beta \vee \alpha \neq \beta)$, which conflicts with our informal insight.

The fact that our argument dealt with $\mathbb{N}^{\mathbb{N}}$ is not really important, if there were a discontinuous function on a subspread of the universal spread, we could apply the above trick just as well.

\subsection{Kripke's Schema and full PEM}

There is another apporach to the non-existence of a discontinuity, basically it is based on the traditional Brouwerian counterexamples. We will, however, use his later approach of the creating subject. The most convenient way is to employ Kripke's Schema:

$$
\text { (KS) } \exists \alpha(\exists x(\alpha(x)=1) \leftrightarrow A)
$$

where $\alpha: \mathbb{N} \rightarrow \mathbb{N}$ and $\sum_{i=0}^{n} \alpha(i) \leq 1$ for all $n$. 
We consider the same function $f$ as in 3.1, with the same approximating sequences $\alpha_{n}$. Moreover, we assume that $f$ is strictly extensional:

$$
(\text { STREXT) } f(x) \# f(y) \rightarrow x \# y
$$

Let $\alpha$ be the Kripke sequence for $P \vee \neg P$ (PEM), i.e., $\exists x(\alpha(x)=1) \leftrightarrow P \vee \neg P$. Define a sequence $\delta$ by

$$
\delta(n)= \begin{cases}0 & \text { if } \forall k \leq n(\alpha(k)=0) \\ \alpha_{k}(n) & \text { if } k \leq n \text { and } \alpha(k)=1\end{cases}
$$

We evaluate $f$ at $\delta: f(\delta)=0 \vee f(\delta) \neq 0$.

If $f(\delta)=0$, then $\forall n(\alpha(n)=0)$, i.e., $\neg(P \vee \neg P)$. Contradiction.

If $f(\delta) \neq 0$, then-because of STREXT- $\delta \# \mathbf{0}$. Hence $\delta=\alpha_{n}$ for some $n$. And therefore $\exists x(\alpha(x)=1)$, which implies $P \vee \neg P$. Since $P$ was arbitrary, we get $\forall P(P \vee \neg P)$. It is an intuitionistic credo that not all propositions are decidable, hence there can be no discontinuity of $f$.

\subsection{The KLST theorem}

We will show in a number of steps that for strictly extensional functions from choice sequences to natural numbers, the undecidability of equality implies continuity, under assumption of Markov's Principle (MP). The proofs are based on a technique introduced by Ishihara [Ishihara 1991]. In fact Ishihara proves a better result than we do here, he gives necessary and sufficient conditions for continuity, weakening Markov's principle considerably. He considers the general case of complete metric spaces; our proof concentrates on the bare essentials, required for metamathematics.

Given the fact from elementary recursion theory that equality between recursive functions is undecidable, the following proof provied a poor man's road to the theorem of Kreisel-Lacombe-Shoenfield-Tsertin (KLST).

We will split the proof in four lemma's.

Let $f: \mathbb{N}^{\mathbb{N}} \rightarrow \mathbb{N}$ be a non-discontinuous function; and let $\lim _{i \rightarrow \infty} \alpha_{i}=\alpha$. Put $f\left(\alpha_{i}\right)=a_{i}, f(\alpha)=a$. We want to show $\lim a_{i}=a$. For the topology of the natural numbers $\lim a_{i}=a$ means just that $a_{i}$ becomes stationary with value $a: \exists i \forall j>i\left(a_{i}=a\right)$.

We can choose the $\alpha_{i}$ such that the paths $\alpha_{i}$ share increasingly longer initial segments with $\alpha$ : there is an increasing sequence $N_{i}$ such that $k>N_{i} \rightarrow \bar{\alpha}_{k} i=\bar{\alpha} i$.

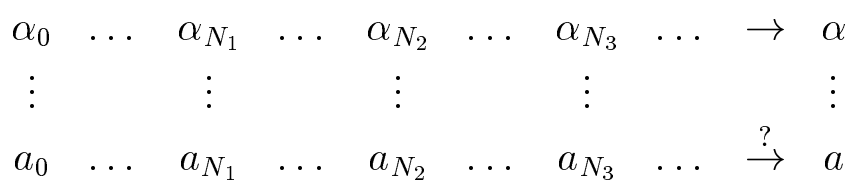


Although the behaviour of the $a_{i}$ is rather mysterious, we know at least something:

Lemma 1 (STREXT) $\forall i\left(a_{i}=a\right) \vee \exists i\left(a_{i} \neq a\right)$

Proof. Define $\beta_{k}= \begin{cases}\alpha & \text { if } \forall j \leq N_{k}\left(a_{j}=a\right) \\ \alpha_{j} & \text { else, where } a_{j} \text { is the first } a_{i} \text { with } a_{i} \neq a\end{cases}$

It is obvious that $\left(\beta_{k}\right)$ converges, say to $\beta$. Put $f(\beta)=b$. The natural numbers have a decidable equality, so $a=b \vee a \neq b$.

If $a=b$, then, by the definition of $\beta_{k}, \forall j\left(a_{j}=a\right)$.

If $a \neq b$, then, by STREXT, $a \# b$, and hence $\exists j\left(a_{j} \neq a\right)$.

Lemma 2 (MP, STREXT) $\lim _{i \rightarrow \infty} a_{i}=a$

Proof. The idea is to go up along the path $\alpha$, checking by Lemma 1 if we have reached the point where the $a_{i}$ 's have become constant.

Define the sequence $\gamma_{i}$, and an auxiliary sequence $\lambda_{i}$ for applying MP.

1.i If $\exists i\left(a_{i} \neq a\right)$, pick the first $a_{i}$ with $a_{i} \neq a$ and put

$$
\left\{\begin{array}{l}
\gamma_{0}=\alpha_{i} \\
\lambda_{0}=1
\end{array}\right.
$$

1.ii Otherwise, put

$$
\left\{\begin{array}{l}
\gamma_{0}=\alpha \\
\lambda_{0}=0
\end{array}\right.
$$

2 Consider the tail sequence $\delta_{k}$ following $\alpha_{i}: \delta_{k}=\alpha_{i+1+k}$. We now apply Lemma 1 to $\left(\delta_{k}\right)$.

2.i If $\exists i\left(f\left(\delta_{i}\right) \neq a\right)$, then put

$$
\left\{\begin{array}{l}
\gamma_{1}=\delta_{i} \text { where } i \text { is the least index with } f\left(\delta_{i}\right) \neq a \\
\lambda_{1}=1
\end{array}\right.
$$

2.ii Otherwise, put

$$
\left\{\begin{array}{l}
\gamma_{1}=\alpha \\
\lambda_{1}=0
\end{array}\right.
$$

3 The general inductive step: let $\gamma_{m}, \lambda_{m}$ be defined. If $\gamma_{m}=\alpha$, then $\gamma_{m+1}=\alpha$ and $\lambda_{m+1}=0$.

Otherwise, we consider the tail sequence $\delta_{k}$ following $\gamma_{m}$. Again, apply Lemma 1 to the sequence $\left(\delta_{k}\right)$. 
3.i If $\exists i\left(f\left(\delta_{i}\right) \neq a\right)$, then put

$$
\left\{\begin{array}{l}
\gamma_{m+1}=\delta_{i} \text { where } i \text { is the least index with } f\left(\delta_{i}\right) \neq a \\
\lambda_{m+1}=1
\end{array}\right.
$$

3.ii Otherwise, put

$$
\left\{\begin{array}{l}
\gamma_{m+1}=\alpha \\
\lambda_{m+1}=0
\end{array}\right.
$$

It is immediate that $\left(\gamma_{i}\right)$ converges, say to $\gamma$. Put $c=f(\gamma)$. Then $c=a \vee c \neq a$.

If $c \neq a$, then, by STREXT, $\gamma \# \alpha$, and hence there cannot be a case (ii), i.e. $\neg \exists i\left(\lambda_{i}=0\right)$. Therefore $\forall i\left(\lambda_{i}=1\right)$. But this implies $\gamma=\alpha$. Contradiction.

So $c=a$. If $\forall i\left(\lambda_{i}=1\right)$, then $f$ is discontinuous (because $f(\gamma)=c \neq f\left(\gamma_{i}\right)$ for all $i$ ), so $\neg \forall i\left(\lambda_{i}=1\right)$, or $\neg \forall i \neg\left(\lambda_{i}=1\right)$. Therefore $\neg \neg \exists i\left(\lambda_{i}=0\right)$, and, by MP, $\exists i\left(\lambda_{i}=0\right)$. This shows that $\lim a_{i}=a$.

Now we have to make the step from sequentially continuous to continuous. The idea here is to consider a suitable enumerable, dense subset of $\mathbb{N}^{\mathbb{N}}$, and to show that $f$ is continuous on it. By sequential continuity, $f$ is then continuous on the whole set. We fix this enumerable subset by taking all sequences which are eventually 0 . They are given by their initial segments preceding the tail of 0 's, and hence they can be enumerated; the enumeration is $\left(\delta_{i}\right)_{i}$. We will prove a lemma, similar to Lemma 1.

Lemma 3 (STREXT) Let $f$ be sequentially continuous. Then

$$
\forall n[\exists \alpha(f(\alpha) \neq n) \vee \forall \alpha(f(\alpha)=n)]
$$

Proof. Consider $\gamma=\lambda i \cdot 1$.

If $f(\gamma) \neq n$, we are done. If $f(\gamma)=n$, we proceed as follows: we consider simultaneously side branches $\gamma_{i}$ of $\gamma$, where $\gamma_{i}=\underbrace{1 \ldots 1}_{i} 000 \ldots$, and $\delta_{i}$ 's.

(1) If $f\left(\gamma_{0}\right)=f\left(\delta_{0}\right)=n$, we put

$$
\beta_{0}=\gamma \text {. }
$$

Otherwise

$$
\beta_{0}=\gamma_{1} .
$$

(2) Let $\beta_{k}$ be defined.

If $\beta_{k}=\gamma$ then we put

$$
\beta_{k+1}= \begin{cases}\gamma & \text { if } f\left(\gamma_{k}\right)=f\left(\delta_{k}\right)=n \\ \gamma_{k+1} & \text { else }\end{cases}
$$


And if $\beta_{k} \# \gamma$, then

$$
\beta_{k+1}=\beta_{k} \text {. }
$$

Clearly $\left(\beta_{k}\right)$ converges, say $\lim \beta_{k}=\beta$. Then $f(\beta)=b$, and $b=n \vee b \neq n$.

If $b \neq n$, then, by STREXT, $\beta \# \gamma$, and hence $\beta=\beta_{i}$ for some $i$, so there are $\gamma_{j}, \delta_{j}$ with $f\left(\gamma_{j}\right) \neq n$ or $f\left(\delta_{j}\right) \neq n$.

If $b=n$, then $\beta=\gamma$ and $\forall i\left(f\left(\delta_{i}\right)=n\right)$. It now immediately follows that $\forall \alpha(f(\alpha)=n)$, as each $\alpha$ is the limit of $\delta_{i}$ 's.

Lemma 4 (MP, STREXT) $f$ is continuous.

Proof. We consider a decreasing sequence of neighbourhoods of some $\alpha$ : $\{\beta \mid \bar{\beta} i=\bar{\alpha} i\}$, and apply Lemma 3 to it.

1. If $\exists \beta(f(\beta) \neq f(\alpha))$, then we put $\gamma_{0}=\beta, \lambda_{0}=0$ for a $\beta$ with $f(\beta) \neq$ $f(\alpha)$.

Otherwise, $\gamma_{0}=\alpha, \lambda_{0}=1$.

2. If $\exists \beta \in \bar{\alpha} 0(f(\beta) \neq f(\alpha))$, then we put $\gamma_{1}=\beta$ for a $\beta$ with $\beta \in \bar{\alpha} 0$ and $f(\beta) \neq f(\alpha)$.

Otherwise, $\gamma_{1}=\alpha$.

3. Assume that $\gamma_{n}$ has been defined.

If $\gamma_{n}=\alpha$, then $\gamma_{n+1}=\alpha, \lambda_{n+1}=1$.

Otherwise, if $\exists \beta \in \bar{\alpha} n(f(\beta) \neq f(\alpha))$, then we put $\gamma_{n+1}=\beta$ for a $\beta$ with $\beta \in \bar{\alpha} n$ and $f(\beta) \neq f(\alpha), \lambda_{n+1}=0$.

$\lim \gamma_{i}=\gamma$. Now, $f(\gamma)=f(\alpha) \vee f(\gamma) \neq f(\alpha)$.

If $f(\gamma) \neq f(\alpha)$, then $\gamma \# \alpha$, hence there are infinitely many $\gamma_{i}^{\prime}$ 's with $f\left(\gamma_{i}\right) \neq f(\alpha)$, hence $f$ is discontinuous. Contradiction.

So $f(\gamma)=f(\alpha)$. Therefore $\neg \forall i\left(\lambda_{i}=0\right)$, and hence $\neg \neg \exists i\left(\lambda_{i}=1\right)$. By MP, we get $\exists i\left(\lambda_{i}=1\right)$. Therefore $\forall \beta \in \bar{\alpha} i(f(\beta)=f(a))$, i.e., $f$ is continuous.

\section{Conclusion}

Together with the main argument in section 2.1, sections 3.1 and 3.2 seem to exhaust our arguments for continuity. Comparing them, Brouwer's original formulation is the superior one. We note that 3.1 and 3.2 yield only negative results (there are no discontinuities), so Brouwer's continuity principle 
is appreciably stronger. We may also note that 3.1 and 3.2 are comparable in strength: the undecidability of identity for choice sequences already eliminates discontinuities, but it also rejects full PEM, hence 3.1 subsumes 3.2, not even considering the fact that 3.1 does not depend on KS.

A few more observations may be added: the argument in 3.1 is perfectly general and applies to any notion of choice sequence (say over the universal tree). Therefore it also applies to the lawlike sequences, which are given by pairs $\left(\alpha(n), R_{n}\right)$, where $R_{n}=R_{0}$ for all $n$, and $R_{0}$ is a law defining $\alpha$. Assuming that identity for lawlike sequences is not decidable, one gets 'negative

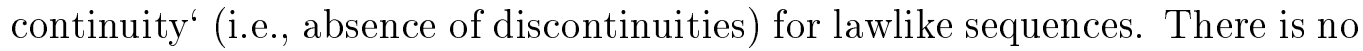
compelling argument for the undecidability of identity for lawlike sequences, although a routine Brouwerian weak counterexample shows that we cannot expect decidability. If one adds Church's Thesis, however, this undecidability can be proved. Brouwer kept clear of continuity for lawlike sequences, but the above arguments show that at least nondiscontinuity can be defended.

\section{References}

[Atten, M. van 1999] M. van Atten. Phenomenology of choice sequences. PhD thesis, Utrecht University, 1999.

[Beeson 1975] M. Beeson. The nonderivability in intuitionistic formal systems of theorems on the continuity of effective operations. Journal of Symbolic Logic, 40:321-346, 1975.

[Beeson 1985] M. Beeson. Foundations of Constructive Mathematics Springer, Berlin. 1985.

[Brouwer 1918] L.E.J. Brouwer. Begründung der Mengenlehre unabhängig vom logischen Satz vom ausgeschlossenen Dritten. Erster Teil, Allgemeine Mengenlehre. KNAW Verhandelingen, 5:1-43, 1918.

[Brouwer 1925] L.E.J. Brouwer. Zur Begründung der intuitionistischen Mathematik I. Mathematische Annalen, 93:244-257, 1925.

[Brouwer 1927] L.E.J. Brouwer. Über Definitionsbereiche von Funktionen. Mathematische Annalen, 97:60-75, 1927.

[Brouwer 1942] L.E.J. Brouwer. Zum freien Werden von Mengen und Funktionen. Indagationes Mathematicae, 4:107-108, 1942. 
[Brouwer 1947] L.E.J. Brouwer. Richtlijnen der intuitionistische wiskunde. Indagationes Mathematicae, 9:197, 1947. Quoted from translation 'Guidelines of intuitionistic mathematics' [Brouwer 1975, p.477].

[Brouwer 1952] L.E.J. Brouwer. Historical background, principles and methods of intuitionism. South African Journal of Science, 49:139-146, 1952.

[Brouwer 1975] L.E.J. Brouwer. Collected works I. Philosophy and Foundations of Mathematics (ed. A. Heyting). North-Holland Publ. Co., Amsterdam, 1975.

[Brouwer 1981] L.E.J. Brouwer. Brouwer's Cambridge Lectures on Intuitionism. Cambridge University Press, Cambridge, 1981.

[Dalen, D. van 1995] D. van Dalen. Hermann Weyl's intuitionistic mathematics. Bulletin of Symbolic Logic, 1:145-169, 1995.

[Heijenoort, J. van 1967] J. van Heijenoort, editor. From Frege to Gödel: A sourcebook in mathematical logic, 1879-1931. Harvard University Press, Cambridge, MA, 1967.

[Hoeven, G. van der 1981] G. van der Hoeven. Projections of lawless sequences. PhD thesis, University of Amsterdam, 1981.

[Husserl 1976] E. Husserl. Ideen zu einer reinen Phänomenologie und phänomenologischen Philosophie. Erstes Buch, volume III/1 of Husserliana. Martinus Nijhoff, Den Haag, 1976.

[Ishihara 1991] H. Ishihara. Continuity and nondiscontinuity in constructive mathematics. Journal of Symbolic Logic, 56:1349-1354, 1991.

[Kreisel 1958] G. Kreisel. A remark on free choice sequences and the topological completeness proofs. Journal of Symbolic Logic, 23:369-388, 1958.

[Kreisel 1967] G. Kreisel. Informal rigour and completeness proofs. In I. Lakatos, editor, Problems in the philosophy of mathematics, pages 138-186. North-Holland, Amsterdam, 1967.

[Kreisel 1968] G. Kreisel. Lawless sequences of natural numbers. Compositio Mathematica, 20:222-248, 1968.

[Troelstra 1977] A.S. Troelstra. Choice Sequences. A chapter of intuitionistic mathematics. Oxford University Press, Oxford, 1977. 
[Troelstra 1983] A.S. Troelstra. Analysing choice sequences. Journal of Philosophical Logic, 12:197-260, 1983.

[Troelstra 1985] A.S. Troelstra. Choice sequences and informal rigour. Synthese, 62:217-227, 1985.

[Troelstra-Dalen 1970] A.S. Troelstra and D. van Dalen. Projections of lawless sequences. In J. Myhill, A. Kino, R.E. Vesley, editors, Intuitionism and proof theory, pages 163-186. North-Holland, Amsterdam, 1970.

[Troelstra-Dalen 1988] A.S. Troelstra and D. van Dalen. Constructivism in Mathematics, I, II. North-Holland Publ. Co., Amsterdam, 1988.

[Veldman 1982] W. Veldman. On the continuity of functions in intuitionistic real analysis. Some remarks on Brouwer's paper: 'Ueber Definitionsbereiche von Funktionen'. Technical Report 8210, Mathematisch Instituut, Katholieke Universiteit Nijmegen, April 1982.

[Weyl 1921] H. Weyl. Über die neue Grundlagenkrise der Mathematik. Mathematische Zeitschrift, 10:39-79, 1921. 